$=12 \mathrm{mg}$ Kaliumpermanganat $=3 \mathrm{mg}$ Sauerstoff $==63 \mathrm{mg}$ organische Substanzen.

Reaktion: 1. Mit Lackmuspapier, das in ein etwa $10 \mathrm{cbm}$ fassendes Porzellanschlchen mit dem betr. Wasser bis zur Hlfte eingetaucht und nach 510 Minuten beobachtet wird. Die meisten Wsser bluen rotes Lackmus. 2. Rosolsurelsung $(0,2$ proz. in 8oproz. Alkohol und durch Barytwasser bis zur rtlichen Frbung neutralisiert) wird in Mengen von 46 Tropfen $50 \mathrm{cbm}$ des zu untersuchenden Wassers zugesetzt: Gelbfrbung = saure Reaktion, Rosa- bis Rotfrbung schwach alkalische oder alkalische Reaktion. 3. Phenolphtalein (1 proz. Lsung in 60proz. Alkohol), Zusatz von 12 Tropfen in $50 \mathrm{cbm}$ Wasser, Rotfrbung zeigt Kalk- oder Alkalihydrat an.

Salpetrige Sure (Nitrite). Ein Reagenzglas bis zu J/4 seines Inhalts mit Wasser angefllt und mit 35 Tropfen 25proz. Phosphorsurelsung und 1012 Tropfen JodZinkstrkelsung versetzt. Blaufrbung zeigt salpetrige Sure an. Je nach ihrer Menge sogleich oder innerhalb einiger Zeit.

Salpetersure. 1. Nachweis mit Diphenylamin: $1 \mathrm{cbm}$ des Wassers wird in einem Porzellanschlchen mit einigen Krnchen Diphenylamin versetzt und in kurzen Zwischenrumen zweimal je 0,5 cbm reine konz. Schwefelsure zugefgt. Positive Reaktion Blaufrbung. 2. Mit Brucin. 3 cbm konz. Schwefelsure werden in einem Reagenzglase mit $1 \mathrm{cbm}$ Wasser tropfenweise gemischt und nach dem Abkhlen einige Krnchen Brucin zugesetzt. Rotfrbung = positive Reaktion.

Schwefelwasserstoff: Am deutlichsten durch den Geruchssinn, weniger scharf durch die Braun-Schwarzfrbung von angefeuchtetem Bleiazetatpapier.

\title{
III. Die Luft.
}

Die atmosphrische Luft ist ein Gasgemenge, bestehend aus 20,7\% Sauerstoff, 78,3\% Stickstoff, etwa $1 \%$ Argon, wechselnden Quantitten (im Mittel etwa $1 \%$ ) Wasserdampf, wechselnden geringen Mengen Kohlensure, sowie stets Spuren von Ozon, Ammoniak, Salpetersure und salpetriger Sure gewhnlich als Ammoniumsalze, zuweilen auch schweflige Sure, Kohlenwasserstoffe u. a. m.

Sauerstoff.

Die Verteilung des Sauerstoffs in der freien Atmosphre ist eine stets gleichmige und zeigt kaum mebare Unterschiede zwischen Wald- und Stadtluft und der in Stdten und Industriegegenden und kaum feststellbare Schwankungen innerhalb bewohnter Rume. Jedenfalls sind Schwankungen im hchsten Falle $0,5 \%$ betragend und hygienisch bedeutungslos. Der Stickstoff der atmosphrischen Luft ist nur als ein indifferentes, den Sauerstoff gleichsam verdnnendes Agens zu betrachten und hat keinerlei 
Funktion mit tierischen oder pflanzlichen Krpern.

Kohlensure.

Der fortlaufenden Produktion der Kohlensure liegen folgende Quellen zugrunde: a) Verbrennung von Brennmaterial besonders in Industriebezirken.

b) Vulkanische Kohlensure, deren unterirdische Ansammlungen aus Bergwerken oder durch Erdspalten ausstrmen, c) Fulnis- und Verwesungsprozesse besonders im gedngten Kulturboden, d) Die Ausatmungsluft der Menschen und Tiere mit einem Gehalt von etwa 4\% der Kohlensure. Der Mensch liefert pro Tag etwa $1000 \mathrm{~g}$ Kohlensure.. Diese von der atmosphrischen Luft aufgenommenen Kohlensuremengen erl; ill : Lustig, Der Arzt als Gesundheitsbeamter. 18

274

eine durchaus gleichmige Verteilung im Freien und auerdem eine ausgiebige Umsetzung einmal durch Lebensprozesse der chlorophyllhaltigen Pflanzen, durch die Niederschlge, die reichlich Kohlensure aufnehmen, und schlielich als die kohlensauren Salze des Meerwassers. Innerhalb bewohnter Rume dagegen kann der Kohlensuregehalt der Luft bei fehlender ausgleichender Verteilung durch Luftbewegung bis zu io pro Mille erreichen. Da gesundheitsschdliche Einflsse durch Kohlensure erst bei einem Gehalt von i\% beginnen und der Tod je nach dem Sauerstoffgehalt bei $1440 \%$ der Luftmenge eintreten kann, ist der gewhnliche Kohlensuregehalt sowohl der Luft als auch der Wohnrume ohne direkten schdlichen Einflu, wenn auch eine Wohnungsluft von mehr als 1 pro Mille Beeintrchtigung des Wohlbefindens auslsen kann.

Bestimmung der Kohlensure. Eine Flasche von bestimmtem Volumen wird mit der zu untersuchenden Luft gefllt und eine abgemessene Menge Barytwasser zugefgt, das sich durch Bildung von Baryunikarbonat trbt. Der durch diese Umsetzung eingetretene Ausfall an Baryumhydrat It sich durch Titrieren mittels einer Normalsure leicht quantitativ ermitteln. Die Differenzzahl gibt einen Mastab fr die Menge Kohlensure, welche in dem abgemessenen Luftquantum enthalten war. Eine approximative Bestimmung der Kohlensure der Luft kann in der Weise geschehen, da durch eine kleine Flasche mit Sodalsung, welche mit einigen Tropfen Phenolphthaleinlsung versetzt und dadurch rot gefrbt ist, die zu untersuchende Luft hindurchgeleitet wird, bis Entfrbung auftritt. Je mehr Luft dazu erforderlich ist, um so geringer ist ihr Kohlensuregehalt. In einen langhalsigen Kolben von etwa 351 Inhalt, dessen Kapazitt vorher durch Ausmessen mit Wasser genau bestimmt war, wird vermittelst eines Blasebalges, der 
mit langem Ansatzrohr versehen ist, durch etwa 50 Ste Luft aus dem Untersuchungsraum eingetrieben. Der Kolben wird mit einer Gummikappe geschlossen, Barometerstand und Temperatur im Raume werden genau festgestellt. Mit einer Pipette, die tief in den Glaskolben eingefhrt wird, It man dann $100 \mathrm{ccm}$ Barytwasser einflieen und schttelt etwa 10 Minutenlang unter allseitigen Drehbewegungen des Kolbens, wobei alle im Kolben vorhandene Kohlensure von der Barytlauge gebunden wird. Die trbgewordene Barv tlauge wird in eine kleine Flasche ausgegossen und luftdicht verschlossen. Nachdem sich ein weier Niederschlag oben abgesetzt hat, pipettiert man von der klaren Flssigkeit $25 \mathrm{ccm}$ ab und titriert mit einer Oxalsurelsung von 2,864 g Oxalsurekristallen in 11 Wasser, auf die vorher das Barytwasser eingestellt war, indem $25 \mathrm{ccm}$ Barytwasser nach. Zusatz von 2 Tropfen iproz. Phenolphthaleinlsung mit der Oxalsure bis zur verschwindenden Rotfrbung titriert werden.

Z. B. waren bei der Titrationsstellung des Barytwassers auf $25 \mathrm{ccm} 24 \mathrm{ccm}$ Oxalsure verbraucht worden, auf $25 \mathrm{ccm}$ des mit der untersuchten Luft geschwenkten Barytwassers nur $22 \mathrm{~cm}$ Oxalsure, so kommen $2 \mathrm{ccm}$ bzw. 8 der ganzen verwendeten $100 \mathrm{ccm}$ auf Rechnung der Kohlensure der untersuchten Luft $=8 \mathrm{mg}$ Kohlensure. Um die Milligramme in $\mathrm{ccm}$ zu verwandeln, mu man erstere durch das Volumengewicht der Kohlensure dividieren, das bei verschiedenen Temperaturen und verschiedenem Luftdruck unterschiedliche Werte hat. War zur Zeit des Versuches die Temperatur 150 bei $760 \mathrm{~mm}$ Barometerstand, so sind die gefundenen $8 \mathrm{mg}$ Kohlensure durch 1,83 zu dividieren (s. Tabelle in einschlgigen Lehrbchern), und man findet so 4,37 ccm Kohlensure. Enthielt die Sammelflasche $3420 \mathrm{ccm}$ Luft, so war der Gehalt der Luft an Kohlen4.37 $\mathrm{x}$

sure $=$------- $==1,28$ pro Mille.

34201000

Approximative Bestimmung, entweder im Lunge-Zeckendorff-Apparat oder in einem weithalsigen Flschchen von etwa $80 \mathrm{ccm}$ Fassungsvermgen, das durch einen doppeltdurchbohrten Kautschukstopfen verschlossen ist. Durch die Einbohrung reicht ein Glasrohr bis zum Boden des Flschchens, whrend an seinem anderen Ende ein Stck Gummischlauch befestigt ist; durch die andere Bohrung wird ein rechtwinkliges gekrmmtes

\section{5}

kurzes Glasrohr gesteckt, dessen ueres Ende durch einen Kautschukschlauch mit einem Gummiballon von etwa $70 \mathrm{ccm}$ Fassung verbunden ist. In dem letzterwhnten Gummischlauch ist ein Lngsschlitz als Ventil angebracht. $2 \mathrm{ccm}$ einer l/10 Normalsodalsung, die durch Phenolphthalein $(0,1 \mathrm{zu} 1000)$ rotgefrbt ist, werden mit $100 \mathrm{ccm}$ destilliertem, ausgekochtem und wieder abgekhltem Wasser verdnnt. Man bringt $10 \mathrm{ccm}$ von dieser Lsung in das eben beschriebene Flschchen. Mit Hilfe des Ballons It man eine Ballonfllung nach der anderen durch die Sodalsung hindurchtreten. Nach jeder frischen Fllung schliet man mit dem Finger den offenen Kautschukschlauch und schttelt das Flschchen eine Minute lang, um alle Kohlensure der Luft zu 
absorbieren, bis die Sodalsung entfrbt ist. Aus der verbrauchten Zahl der Ballonfllungen It sich der Kohlensuregehalt der Luft annhernd berechnen. Man gebraucht im Mittel bei einer Luft von 0,3 pro Mille Kohlensure 48 Ballonfllungen, bei 0,6 pro Mille 21 Ballonfllungen, bei 1 pro Mille neun Ballonfllungen (s. Tabelle in Lehrbchern). Ist der Kohlensuregehalt der Luft hher als 1 pro Mille, so wird der Versuch mit einer doppelt so starken Sodalsung $(=2 \mathrm{ccm}$ Stammlsung $=50 \mathrm{ccm}$ Wasser) wiederholt, fr diese Verdnnung ist eine weitere Umrechnungstabelle vorhanden.

Ozon- und Wasserstoffsuperoxyd stellen die sog. oxydierende Kraft der Luft dar. Am wenigsten Ozon beobachtet man bei trockenen Nord- und Nord west winden im Herbst, die grten Mengen im Frhjahr bei feuchter bewegter Luft nach Gewittern und Schneefall. Eine Steigerung des Ozongehalts ist in WIdern, am Meere, auf Bergen zu beobachten, in den meisten greren Stdten ist in der Straenluft sowie in bewohnten Rumen Ozon nicht nachweisbar. Eine erhebliche hygienische Bedeutung It sich dem Ozongehalt der Luft nicht nachweisen. Ebensowenig dem atmosphrischen Wasserstoffsuperoxyd. Kohlenoxydgas ist in der freien Atmosphre in nachweisbaren Mengen nicht haltbar, im Wohnraum kann es in solchen Mengen Vorkommen, da Vergiftungen entstehen, z. B. bei ausstrmendem Leuchtgas oder Heizgasen.

Untersuchung der Luft auf Kohlenoxyd: Die verdchtige Luft wird wie bei der Kohlensurebestimmung in einen Glaskolben eingetrieben und mit $20 \mathrm{ccm}$ einer 20proz. Blutlsung 1/i Stunde lang geschttelt. Der Nachweis von Kohlenoxyd erfolgt: 1. chemisch: $15 \mathrm{ccm}$ iproz. Tanninlsung werden in Mengen zu je $5 \mathrm{ccm}$ der fraglichen Blutlsung und einer gleichstarken normalen Blutlsung zugesetzt und geschttelt. Bei Kohlenoxyd-Blut entsteht ein Niederschlag, der nach 12 Stunden brunlichrot wird, beim Kontrollblut dagegen ein graubrauner Niederschlag. Oder $5 \mathrm{ccm} 20 p r o z$. Ferrozyankaliumlsung und $1 \mathrm{ccm} 33$ proz. Essigsure werden zu je $10 \mathrm{ccm}$ der fraglichen Blutlsung und einer gleichstarken normalen Blutlsung zugesetzt und geschttelt. Bei Kohlenoxydblut bildet sich alsbald ein rotbrauner, beim Kontrollblut ein graubrauner Niederschlag.

2. spektroskopisch: Beide Blutlsungen werden bis zu blaroter Farbe ca. 200- bis $300 f a c h$ verdnnt und im Spektroskop verglichen. Kohlenoxydblut gibt zwei Absorptionsstreifen im Gelb (zwischen den Linien D und E).

Das Vorkommen von Gasen, wie Chlor, schwefliger Sure, salpetriger Sure, in Mengen, die die Gesundheit gefhrden, wird hchstens in Fabrikrumen beobachtet. In der Wohnungsluft hufiger schweflige 
Sure. Der Nachweis erfolgt dadurch, da man grere Mengen verdchtiger Luft durch Kalilauge streichen It und die absorbierten Gase nach den blichen Methoden titrimetrisch bestimmt.

Bestimmungen der schwefligen Sure in der Luft: Mittels eines Aspirators wird eine bestimmte Luftmenge durch eine Peligot sehe Rhre mit $20 \mathrm{ccm} \mathrm{n} / 50-J o d l s u n g$

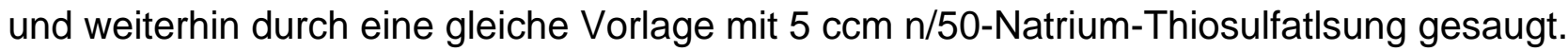

Nach dem Durchgleiten giet man beide Lsungen in ein Becherglas zusammen, splt beide Vorlagen mit destilliertem Wasser nach und in das Becherglas hinein, setzt einen Tropfen Strkelsung als Indikator zu und titriert bis zur Entfrbung. Berechnung:

Wren nach dem Durchsaugen der Luft zur Titration der $15 \mathrm{ccm}$ Jodlsung $\{20 \mathrm{ccm}$ Jodlsung $5 \mathrm{ccm}$ Natrium-Thiosulfatlsung) nicht 75 , sondern nur $10 \mathrm{ccm} \mathrm{n/50Natrium-}$ Thiosulfatlsung gebraucht worden, so wren $5 \mathrm{ccm}$ der Jodlsung durch schweflige Sure gebunden worden. Da $1 \mathrm{ccm}$ der Jodlsung $=180 \mathrm{mg}$ schwefliger Sure entspricht, entprechen $5 \mathrm{ccm}=640 \mathrm{mg}$ schwefliger Sure. Die durchgesaugte Luftmenge berechnet sich auf enthielt also 0,64 $\mathrm{g}$ schweflige Sure.

belriechende Gase, wie Schwefeiwasserstoff,Merkaptane, flchtige Fettsuren u. a. m., die bei Fulnisprozessen usw. aus Aborten, Gruben und Kanlen, durch Ausdnstung der Menschen, Kchendnste usw. entstehen, kommen weder in der reinen Luft noch in den Wohnungen kaum in solchen Mengen vor, da Giftwirkungen entstehen knnen, dagegen sind Schdigungen dieser heftig wirkenden Gifte durch ihre Ansammlungen in Kanlen oder Abortgruben wiederholt beobachtet worden. Ebensowenig haben belriechende Ausdnstungen der Menschen gesundheitsschdigende Wirkungen auer der Beeintrchtigung einer durch Ekel behinderten tiefen Atmung. Die bei hoher Anhufung von Ausatmungsstoffen in berfllten Rumen beobachteten Gesundheitsstrungen treten nur bei hheren Temperaturen und erhhter Luftfeuchtigkeit auf und sind lediglich durch Wrmestauung bedingt.

Luftstaub.

Die in der Luft suspendierten Elemente stellen grere Staubpartikelchen, $\mathrm{Ru}$, Sonnenstubchen und Mikroorganismen dar. Grob sichtbarer Staub, gewhnlich der Bodenoberflche entstammend, kann in $1 \mathrm{ccm}$ Luft 
in Mengen von 0,225 mg vorhanden sein, und zwar im Sommer in den hchsten, im Frhjahr in den niedrigsten Mengewerten. Er besteht zu 2/33/4 aus mineralischen Substanzen, der Rest aus organischem Detritus mit vielen toten und lebensfhigen Keimen.

Rauch und Ru enthalten Kohlenteilchen bzw. Kohlenwasserstoffe, sowie Verbrennungsgase, die bei hinreichender Luftfeuchtigkeit zu dicken Nebelschichten beitragen knnen und durch Behinderung der Sonnenstrahlung und Tageshelligkeit wohl Belstigungen der Menschen hervorrufen, aber keine akuten gesundheitsschdigende Auswirkungen haben. Die gewhnlich in der Luft erst durch einfallende Sonnenlichtstrahlen sichtbaren Sonnenstubchen sind so leicht, da sie selbst bei ruhiger Luft sich nicht vollstndig absetzen und bis zu den grten Hhen der Atmosphre verbreitet sind. Sie bestehen in der Regel aus organischem Detritus u. .

Der gesamte Luftstaub wird quantitativ bestimmt, indem man die Luft durch ein Glasrohr mit Watteeinlagerung aspiriert und die Gewichtszunahme bestimmt. Sollen Rumengen allein festgestellt werden, so saugt man Luft in grerer Menge PA cbm) durch angefeuchtetes Filtrierpapier, grbere Rupartikel lassen sich in mit I ausgestrichenen Schalen in horizontaler oder vertikaler Richtung auffangen. Die Schwrzung der Aufnahmeflchen, kolometrisch abgeschtzt, zeigt das Ergebnis an. Die mikroskopische Untersuchung des Luftstaubs wird vermittels einer Glasplatte vorgenommen, auf der durch einen klebrigen berzug der Staubgehalt der Luft bei bestimmter Geschwindigkeit des Luftstromes sich niederschlgt. Zur Zhlung der Staubteilchen wird das Verfahren

\section{7}

von Aitken benutzt, nach welchem die kleinsten Staubteilchen als Wasserkondensationskerne durch eine mit feiner Teilung versehene Glasplatte gezhlt werden.

Mikroorganismen.

Mit der Entfernung vom Festlande nach dem Meere zu und von der Erdoberflche in die Hhe nimmt die Keimzahl der Luft ab, gleichwie auch der Keimgehalt der Luft im Vergleich zu Wasser und Boden relativ gering ist. Die Keimarmut der Atmosphre kann wohl darin gesucht werden, da die Keime bald zu Boden sinken und nur unter besonderen Umstnden wieder in die Luft aufgewirbelt werden. Wesentlich dafr ist, ob die auffangende Flche na oder trocken ist, ferner erhebt sich der Staub leichter von glatten als von rauhen Unterlagen. Da die Keime durch auf- und absteigende Luftstrme in die Atmosphre verschleppt werden, enthlt die Luft im Winter durchschnittlich weniger Keime als 
im Sommer. Die hauptschlichsten Mengen der in der Luft befindlichen Keime haften an den mineralischen Staubpartikelchen sowie an den grberen und feineren Fasern, welche sich von Kleider- und Mbelstoffen losisen, daher ist der grob sichtbare Staub im allgemeinen keimreicher als die feinsten Staubelemente und zum Weitertransport der Bakterien weniger geeignet als die feinsten Feuchtigkeitstrpfchen. Der Gattung nach gehren die Luftkeime zu den Saprophyten der Boden- und Wasserflora, whrend die pathogenen Keime in der Luft die ntigen Existenzbedingungen nicht finden und nur vorbergehend vorhanden sind. Unter den Luftkeimen berwiegen der Zahl nach hauptschlich Kokken, daneben sporenbildende Bakterien und Sarcinen, in den hheren Luftschichten finden sich berwiegend die Spro- und Schimmelpilze.

Bakteriologische Keimzhlung der Luft. i. Nach Hesse: Ein Glasrohr von $70 \mathrm{~cm}$ Lnge und 3,5 cm Weite wird mit Nhrgelatine beschickt in der Weise, da sie nach dem Eistarren die ganze Glaswand in dnner Schicht auskleidet. Durch dieses Glasrohr It man 10201 Luft langsam durchstrmen, etwa 11 in 24 Minuten. Die auf der Gelatine entwickelten Keime werden gezhlt und auch qualitativ weiter untersucht. 2. Nach Petri: In ein kurzes, etwa $2 \mathrm{~cm}$ weites Glasrohr wird ein Stck Drahtnetz eingeklemmt, darauf kommt eine etwa $3 \mathrm{~cm}$ dicke Schicht grober Sand von 0,4 mm Korngre, dann wieder ein Drahtnetz. Das so hergestellte Filter wird sterilisiert, mit einem krftigen Aspirator verbunden und die zu untersuchende Luft in raschem Strome durchgesogen. Das Filter hlt alle Keime zurck, die mit dem Sand und dem Drahtnetz in Gelatine oder Agar gebracht werden und zur Entwicklung gelangen.

Die Luftfeuchtigkeit.

Die Luft vermag bestimmte Mengen des bei dem Verdunsten des Wassers gebildeten Wasserdampf aufzunehmen, und zwar vergrert sich dies Aufnahmevermgen mit steigender Temperatur. Fr jeden Temperaturgrad ist die Aufnahmefhigkeit der Luft fr Wasserdampf scharf begrenzt. Diese maximale Feuchtigkeit zu jedem Temperaturgrad ist aus der sog. Spannungstafel abzulesen. In der Regel ist aber die Luft nicht mit Wasserdampf gesttigt, sondern enthlt nur geringere Mengen = absolute Feuchtigkeit, $\mathrm{d}$. h. diejenige Menge Wasserdampf, die pro cbm Luft augenblicklich in der Luft enthalten ist. Die relative

\section{8}

Feuchtigkeit gibt die vorhandene $=$ absolute Feuchtigkeit in Prozenten der fr die bestehende Temperatur mglichen maximalen Feuch.

$r . \wedge^{\wedge} .100 \mathrm{X}$ abs. Feuchtigkeit 


\section{Feuchtigkeit}

Das Sttigungsdefizit ist die Differenz zwischen maximaler und absoluter Feuchtigkeit. Der Taupunkt ist diejenige Temperatur, fr welche die Luft mit der augenblicklich vorhandenen Feuchtigkeit gesttigt ist, bzw. maximale Feuchtigkeit = absolute Feuchtigkeit.

Die hygienische Bedeutung der Luftfeuchtigkeit liegt darin, da von ihr die Wasserdampfabgabe und durch Vermittlung dieser die Wrmeabgabe vom Krper abhngig ist. Whrend ein berschu der Wasserdampfabgabe vom Krper hchstens zu vermehrtem Durstgefhl fhrt bzw.

zur Trockenheit exponierter Schleimhute ist eine Hemmung der Wasserdampfabgabe mit einer Wrmestauung verbunden, die $u$. U. lebensgefhrlich werden kann. Bei mittleren Temperaturen von 1820 erscheinen $4050 \%$ relative Feuchtigkeit am gnstigsten, bei hheren Temperaturen 3040. Extrem niedrige Feuchtigkeitsprozente bedingen durch Erleichterung der Wrmeabgabe Wohlbehagen; bei niederer Temperatur sind sie ohne erhebliche Wirkung.

Bestimmung der Luftfeuchtigkeit, i. Durch Wgung des Wasserdampfes, welcher aus einem gemessenen Luftquantum durch Kalzium-Chlorid oder Schwefelsure absorbiert ist. 2. Das Kondensationshygrometer bestimmt den Taupunkt, mit dem aus beigegebener Tabelle die absolute Feuchtigkeit zu entnehmen ist. 3. Das Haarhygrometer beruht auf der Verlngerung entfetteter Haare bei steigender relativer Feuchtigkeit, die durch einen damit in Verbindung stehenden Zeiger auf einer Skala angezeigt wird. 4. Psychrometer. Zwei Thermometer, von welchen die Kugel des einen von mit Wasser befeuchtetem Mull umhllt ist. An dem feuchten Thermometer wird um so mehr Wasser verdunstet, je trockener die Luft und je niedriger der Barometerstand; durch die Wasserverdunstung zeigt das feuchte Thermometer eine um so niedrigere Temperatur gegenber dem trockenen Thermometer an, je austrocknender die Luft wirkt.

Hat das feuchte Thermometer seinen tiefsten Stand erreicht, so berechnet man aus der Temperatur des trockenen und des feuchten Thermometers die absolute Feuchtigkeit mit Hilfe von Tabellen. Genauere Resultate liefert das Amannsche Aspirationspsychrometer oder ein Schleuderpsychrometer. Die Luftbewegung ist abhngig von den Verhltnissen des Luftdrucks bzw. von der Temperatur. Ihre genauen Messungen werden durch Anemometer ausgefhrt, und zwar entweder durch Feststellung des Winddruckes, oder bei dem Flgel-Anemometer entnimmt man die Geschwindigkeit aus der Zahl der Umdrehungen eines Rotationsapparates.

Die Messung des Luftdrucks fr hygienische Zwecke geschieht wie allgemein durch das Quecksilberbarometer oder Holosteric-Aneroid-Barometer, wobei nach jeder Ablesung eine Reduktion der Barometerangabe auf o mit Hilfe von Tabellen vorgenommen wird. Die Wrmemessung der Atmosphre erfolgt am genauesten durch das Schleuderthermometer, 
d. $h$, durch ein gewhnliches Thermometer, welches an einer $1 \mathrm{~m}$ langen

Schnur einige Male im Kreise geschwungen wird oder durch das Amannsche

Aspirationsthermometer.

Fr groe Kltegrade kommen Weingeistthermometer in Anwendung.

Bei der gewhnlichen Messung durch Quecksilberthermometer mu dasselbe gegen die Strahlung vom Boden und erwrmter Hauswnde geschtzt werden, am besten dadurch, da man das Thermometer an der Nordwand des Hauses mindestens $4 \mathrm{~m}$ ber dem Boden und so in einem Gehuse anbringt, da nur die zutretende Luft auf das Thermometer einwirkt.

Bei der Sonnenstrahlung, von der die auf die Erde gelangende Wrme ausschlielich herrhrt, unterscheidet man 1. die langwelligen roten Wrmestrahlen. 2. die gelben Helligkeitsstrahlen, 3. die kurzwelligen blauvioletten (photochemischen) und ultravioletten

\section{9}

Strahlen. Die Messung der Wrmestrahlen erfolgt durch das Pyrheliometer und Aktinometer, die der Helligkeitsstrahlen durch das Photometer, die der kurzwelligen Strahlen vermittelst Bromsilberpapier.

\section{Stdtebau.}

Fr die Vergrerung von Stdten oder die Anlegung von Ortschaften werden als allgemeine Grundlagen bestimmte Bebauungsplne aufgestellt, in denen fr eine krzere oder Inger gefate Zukunft die Baubedrfnisse der Wohnungswirtschaft, der ffentlichen Gebude, fr Gewerbe und Industrie projektiert sind. Der Umfang des Bebauungsplanes ist naturgem auf rtliche Begrenzungen und Beschaffenheit des vorliegenden Gelndes angewiesen, dessen Ausnutzung aus wirtschaftlichen, bau- und verkehrstechnischen Bedingungen nicht zu umgehen ist. Es ist daher die Aufgabe der Hygiene, die gesundheitlichen Anforderungen mit diesen Interessen in Einklang zu bringen und besondere rtliche hygienische Mistnde zu beseitigen.

Bezglich der Oberflchengestaltung des Gelndes ist zu bercksichtigen, da geringe Neigung des Terrains oder vorhandene Mulden- und Bodenfalten zu oberflchlichen Wasseransammlungen und Feuchtigkeit Anla geben, da enge Tler an stagnierender Luft und feuchtem Boden zu leiden haben, da exponiert gelegene Pltze z. B. auf Bergrcken mit heftigen Windstrmungen, vegetationslose Hochplateaus extremen Temperaturschwankungen ausgesetzt sind. Im berschwemmungsgebiet eines Flusses gelegenes Gelnde ist der Einwirkung von Hochwasserschden zu entziehen. Vorhandene Wasserlufe sollen nicht unmittelbar in den Wohnhuserblock zu liegen kommen, sondern sind zu ihref leichteren Reinhaltung mit 
Parkanlagen und freien PItzen zu vereinigen.

Was die Bodenart anbetrifft, so ist die Gesteinsformation weniger von hygienischer Bedeutung als die Beschaffenheit der obersten Bodenschichten, ob dieselben in den letzten Jahrzehnten durch knstliche Aufschttung oder im Laufe von Jahrhunderten oder Jahrtausenden durch natrliche Einflsse entstanden sind. Der Stand des Grundwassers und seine Schwankungen mssen bekannt sein, ein Steigen seines Spiegels in die Bewohnungszone fhrt neben der allmhlich aufsteigenden Durchfeuchtung der Wohnhuser zur Verschlechterung des Grundwassers infolge Verunreinigung mit hauswirtschaftlichen und gewerblichen Abwssern.

Die hygienischen Anforderungen beziehen sich weiterhin auf die Wasserversorgung, Fernhaltung von durch Lrm und Ausdnstung als gesundheitsschdigend anzusehenden ffentlichen und Privatbetrieben (Schlachthfe, Abdeckereien, Viehhfe, gewerbliche und industrielle Anlagen) aus reinen Wohngegenden, ferner auf die Frsorge fr Licht und Luft.

In der Art der Ansiedlung unterscheidet man offene, halboffene und geschlossene Bauweisen.

Die offene Bauweise ist das Gebiet des Ein- oder Zweifamilienhauses, in Grten und Hfen gelegen, allseitig von Licht und Luft umsplt.

Diese offene Bauweise eignet sich aber nur fr reine Wohngebude auerhalb der verkehrsreichen Stadtteile und Gewerbeviertel, da der Lrm und Staub in die offene Anlage ungehindert eindringen kann. Ihre zweckmige Durchfhrung kommt in den sog. Villenvierteln und Gartenstdten besonders zur Geltung. Whrend das Villenviertel, die Domne 